\title{
Anesthetic management of an undiagnosed advanced extrauterine pregnancy under combined spinal-epidural anesthesia
}

\author{
Arun Kalava ${ }^{1 *}$, Simon Mardakh', Jonathan Weinberg' ${ }^{1}$, Joel Yarmush'1, Joseph Schianodicola', Khaja Ahmed \\ Correspondence: arunkalava@yahoo.com \\ 'Department of Anesthesiology, New York Methodist Hospital, Brooklyn, New York, USA.
}

\begin{abstract}
Advanced extrauterine (abdominal) pregnancy is extremely rare. Management of such a pregnancy invariably involves a laparotomy under general anesthesia and has never been reported to have been performed in its entirety under neuraxial anesthesia. We report a case of a 26- year-old multigravida who presented with severe abdominal pain at 32 weeks and 1 day gestational age and on laparotomy was found to have an undiagnosed extrauterine pregnancy. We believe this is the very first reported case of advanced extrauterine pregnancy, diagnosed at laparotomy, performed under combined spinal-epidural anesthesia and successfully managed in its entirety, without conversion to general anesthesia. The postoperative period was uneventful, with both mother and neonate being discharged 3 days after surgery. Through this case we discuss the management and outcome of this rare presentation performed successfully under neuraxial anesthesia.
\end{abstract}

Keywords: Extrauterine pregnancy, combined spinal epidural anesthesia, anesthetic management

\section{Introduction}

The reported incidence of abdominal pregnancy is 10.9 per 100,000 live births and 9.2 per 1000 ectopic pregnancies in the United States [1], with higher incidence seen in developing nations [2]. Management of such a pregnancy depends on the gestational age and invariably involves surgical intervention i.e., laparoscopy or laparotomy. General anesthesia with tracheal intubation is preferred for a surgery involving an extrauterine pregnancy, as it is frequently complicated by extensive hemorrhage. In the recent decades maternal mortality and morbidity from pulmonary aspiration (incidence 1:500 to 1: 400 for obstetric patients versus 1:2000 for all patients) and failed endotracheal intubation (incidence of 1: 300 to 1:250 [35] versus 1:2000 for all patients) have been the primary motivators for transition towards greater use of neuraxial anesthesia instead of general anesthesia in cesarean delivery. Further, difficult intubation is relatively common (65\% of total difficult intubations) in patients undergoing emergency cesarean section [6]. Rice T and Bowser C reported a case of advanced extrauterine pregnancy at 34 weeks that was diagnosed at laparotomy under spinal anesthesia, with the patient having to be immediately anesthetized and intubated for the duration of the surgery [7]. To our knowledge, it has never been reported of having been performed in its entirety under combined spinal-epidural (CSE) anesthesia.

\section{Case report}

A 26-year-old, American Society of Anesthesiologists (ASA) physical status II E, Gravida 7, Para 2, with 2 prior Cesarean sections, history of placenta previa, and who had prenatal care elsewhere, presented to the emergency department at a 32 week and 1 day gestational age with severe abdominal pain. She was on prenatal vitamins and had no known drug allergies. She denied smoking cigarettes and consuming alcohol. A bedside ultrasound done by the obstetrician showed a fetus in transverse lie, with a heart rate of 150 /minute and placenta lying below the fetus. A diagnosis of acute abdomen was made and the patient was scheduled for an emergency exploratory laparotomy to rule out placental abruption vs. uterine rupture with possible placenta accreta.

On pre-anesthetic evaluation, we found a young anxious woman, who was 64 inches tall and weighed 192 pounds with a BMl of $33 \mathrm{~kg} / \mathrm{m}^{2}$. Her blood pressure was $93 / 63 \mathrm{~mm}$ $\mathrm{Hg}$ (mean arterial pressure of 73), and heart rate was 97/ minute. On airway assessment, the patient had adequate mouth opening, no loose teeth, caps or crowns and the Mallampati grade was III. There were no masses palpable in the neck, she had good flexion/extension of the neck and the temporo-mandibular distance was 3 fingers breadth. She was afebrile, heart and lungs were clear to auscultation. There was severe generalized tenderness of the abdomen with no signs of peritonitis. Bilateral 
pedal edema was noted. Preoperative laboratory workup revealed leucocytosis (WBC $\left.=15.2 \times 10^{9} / \mathrm{L}\right)$, anemia $(\mathrm{Hb}=9$ $\mathrm{gm} / \mathrm{dL}$ ), and a normal platelet count.

CSE anesthesia was planned for the exploratory laparotomy as the patient was neither in a state of shock nor was hypotensive. An informed consent was obtained after the risks of extensive bleeding, possible blood transfusion, general anesthesia and hysterectomy were explained to the patient.

Patient had two 18 gauge intravenous lines inserted and 1.5 liters of lactated ringer's USP was given preoperatively. In the operating room standard ASA monitors were applied and oxygen $\left(\mathrm{O}_{2}\right)$ was delivered at $3 \mathrm{~L} /$ minute via nasal cannula. A left radial arterial line was placed for invasive blood pressure monitoring. Patient's vitals before start of anesthesia were: blood pressure $=111 / 81 \mathrm{~mm} \mathrm{Hg}$, pulse $97 /$ minute, $\mathrm{O} 2$ saturation of $99 \%$ on $3 \mathrm{~L}$ of $\mathrm{O}_{2}$.

A CSE was performed with the patient in a sitting posture, and a mixture of $1.8 \mathrm{~mL}$ of $0.75 \%$ bupivacaine, $100 \mathrm{mcg}$ of epinephrine, $25 \mathrm{mcg}$ of fentanyl and $100 \mathrm{mcg}$ of morphine was used for subarachnoid block. A slightly higher spinal dose was used as we anticipated a longer than normal surgical time. Patient was then made to lie supine with a left tilt position to avoid aortocaval compression. Vital signs immediately after CSE were, blood pressure $=100 / 66$ $\mathrm{mm} \mathrm{Hg}$, pulse $=106 /$ minute, $\mathrm{O}_{2}$ saturation of $100 \%$ on 3 $\mathrm{L}$ of $\mathrm{O}_{2}$. A Foley catheter was placed and a body warmer was applied. Cefazolin $2 \mathrm{gm}$ IV was administered just prior to skin incision.

On opening the abdomen with a Pfannenstiel incision, the surgeon noted bluish coloration of the anterior fundal uterine wall, with an amniotic sac with fetus, and the membranes entirely in the abdominal cavity with placenta adherent to the anterior fundal uterine wall. The membranes were ruptured and a female infant found to be in transverse lie, was delivered via breech presentation, 11 minutes after incision. The infant weighed 1304 grams and was 16.5 inches with Apgar scores of 7 and 8 at one and five minutes respectively. Extensive hemorrhage was noted after the uterus was exteriorized and a supracervical hysterectomy was performed for placenta percreta. Intraoperatively, patient's blood pressure was managed with lactated ringer's USP and 6\% Hetastarch USP (Hextend, Hospira, Inc., Lake Forest, IL 60045) along with 100-200 mcg boluses of phenylephrine. Patient also received 2 units of blood intraoperatively $(\mathrm{Hb}=7.8 \mathrm{~g} / \mathrm{dL})$ and two additional units postoperatively. Estimated blood loss during the surgery was 2 liters. Patient was awake throughout the surgery, which lasted 111 minutes. There were no clinical signs suggestive of disseminated intravascular coagulation and also the obstetrician achieved good hemostasis. At the end of the surgery, the epidural catheter was removed and patient was transferred to post-anesthesia care unit with vital signs of blood pressure $=121 / 70 \mathrm{mmHg}$, pulse $=72 /$ minute, respiratory rate of $14 /$ minute and $\mathrm{O}_{2}$ saturation of $100 \%$.

Her postoperative period was uneventful and she was discharged 3 days after surgery. Surgical pathology confirmed placenta percreta and extensive extrauterine growth of the chorionic disc on the anterior aspect of the uterus and reported that there appears to have been a uterine rupture, but potentially quite remote to delivery as the overwhelming majority of the placental gestational sac appears to have been extrauterine; the uterine cavity itself appears small, although the specimen was bivalved and the site of rupture appears to have been anterior. The above report suggested that the fetus migrated through a uterine rupture into the abdomen with the placenta and adhered to serosa.

\section{Discussion}

Abnormal placentation is of 3 types:

1. Placenta accreta: where placenta invades the myometrium without penetration into the entire thickness of the muscle.

2. Placenta increta: Placenta further extends into the myometrium, penetrating the muscle and

3. Placenta percreta: Placenta penetrates the entire myometrium to the uterine serosa.

Extrauterine pregnancy is a type of pregnancy, where the placenta is implanted in the peritoneal cavity exclusive of tubal, ovarian, or intraligamentary implantations [8]. The most frequent symptom encountered in an advanced extrauterine pregnancy is abdominal pain (100\%), and the commonest physical findings are abdominal tenderness (100\%) and an abnormal fetal lie (70\%) [9]. Diagnosis using an ultrasound, with clinical correlation has $50 \%$ estimated success rate [10]. An MRI scan is helpful in diagnosis [11], but is of limited use in an emergency. Hence, despite advances in technology, only 1 out of every 9 women who reached the hospital alive has an accurate preoperative diagnosis of abdominal pregnancy [1] and it was no different in our case, as it was diagnosed intraoperatively.

Maternal mortality is about 8 times higher among women who present with abdominal pregnancies compared with other forms of ectopic pregnancy and ranges from 0 to $30 \%$ [12]. Majority of the reported deaths are due to maternal hemorrhage and postpartum sepsis [9]. Placental removal is recommended if the blood supply to the placenta can be controlled. In the event of any doubt about hemorrhage control, it is advised to leave the placenta undisturbed. Methotrexate has been used in the past to expedite degeneration of the trophoblastic tissue in the residual placenta [9], while some have condemned its use [13]. Secondary hemorrhage, disseminated intravascular coagulation, postpartum preeclampsia [14], intestinal obstruction/perforation/fistula formation, sepsis and abscess formation are all complications that can occur from a retained placenta [15]. Placenta in this case was attached in its entirety to the anterior fundal uterine wall and hence 
a decision to perform a hysterectomy was made, with an excellent outcome.

In 1993, Stevens CA's analysis of 1,161 cases of abdominal pregnancies since 1809 showed a $21.4 \%$ rate of malformations and deformations among infants [16] and the perinatal mortality is worse for the fetus being 45 to $90 \%$ [17]. In our case, the outcome was excellent as the baby had no fetal anomalies and had an intact amniotic sac with sufficient amniotic fluid at the time of delivery. On a telephonic follow up, a year later with the patient, the child was healthy and reportedly had no developmental delays. General anesthesia is usually preferred when major intraoperative hemorrhage is anticipated. Hughes et al., described the anesthetic management of an abdominal pregnancy, diagnosed preoperatively, under general anesthesia [11]. With an anticipation of extensive blood loss, our anesthetic team was prepared to manage intraoperative hemorrhagic complications that would arise from undiagnosed abnormal placentation or uterine rupture needing emergent hysterectomy. With the experience of having had performed obstetrical hysterectomies under neuraxial anesthesia and with our patient being hemodynamically stable, with no obvious signs of shock or hypotension, a decision on performing the surgery under CSE was made instead of general anesthesia. CSE also allows for a reduced spinal dose and the epidural catheter serves as a backup to extend the duration of neuraxial block if needed. Our anesthetic team was also anticipating the likely conversion from neuraxial to general anesthesia given the abnormal placentation on the ultrasound. The keys to success in our particular case were:

1. Intravenous preloading prior to performance of CSE anesthesia.

2. Starting invasive monitoring i.e., a radial arterial line.

3. Cross-matched blood made immediately available along with rapid transfusion systems for their administration.

4. Placental attachment to the anterior uterine fundus made catastrophic hemorrhage less likely as would have been the case if it was attached to bowel or mesentery as even a partial or complete separation from those sites carries a risk of exsanguination [18].

5. Aggressive intraoperative fluid management, which included use of lactated ringer's USP, $6 \%$ Hetastarch and blood transfusion.

6. Use of vasoactive drugs like phenylephrine as needed and availability of other vasopressors like ephedrine, epinephrine and dopamine.

We suggest that cases with placental attachment localized to the anterior uterine fundus diagnosed prior to operation may be selectively managed under CSE, with general anesthesia as back up.

Neuraxial anesthesia is routinely used for obstetric surgical emergencies as it has less risk of failed intubation and aspiration and thus reducing maternal morbidity and mortality [19]. Any abnormal placentation carries the risk of torrential hemorrhage and we believe that select cases can be performed under neuraxial anesthesia with invasive monitoring (arterial lines and/or central venous catheters) and aggressive volume resuscitation. Management of placenta accreta, that needed a cesarean hysterectomy have been successfully performed under neuraxial anesthesia $[20,21]$. Thus, anesthetic regimen of an obstetric emergency should always be tailored to the patient's requirements intraoperatively, with the team being ready to manage massive hemorrhage and transition to general anesthesia if necessary.

\section{Conclusion}

Obstetric emergencies, where difficult intubation and risk of pulmonary aspiration are high, can be managed under neuraxial anesthesia if the patient is hemodynamically stable at presentation and remains relatively stable intraoperatively, not requiring massive transfusion, as was possible in our case. But, this approach cannot be justified in other situations with extensive hemorrhage and massive blood transfusion, where general anesthesia would be inevitable.

\section{Competing interests}

The Authors' declare that they have no competing interests.

\section{Authors' contribution}

AK: Prepared the manuscript, SM: Involved in the case, JW: Prepared the manuscript, JMY: Prepared the manuscript, JS: Approved the manuscript, KYA: Involved in the case.

Acknowledgments

We would like to thank the team of obstetricians, nurses and technicians who were involved in successfully managing this patient.

Publication history

Received: 07-Sep-2012 Revised: 07-Oct-2012

Accepted: 16-Oct-2012 Published: 03-Nov-2012

\section{References}

1. Atrash HK, Friede A, Hogue CJ: Abdominal pregnancy in the United States: frequency and maternal mortality. Obstet Gynecol 1987, 69:333-337. | Article | PubMed

2. Sunday-Adeoye I, Twomey D, Egwuatu EV, Okonta PI: A 30year review of advanced abdominal pregnancy at the Mater Misericordiae Hospital, Afikpo, southeastern Nigeria (1976-2006). Arch Gynecol Obstet 2011, 283:19-24. | Article | PubMed

3. Hawthorne L, Wilson R, Lyons $G$, Dresner M: Failed intubation revisited: 17-yr experience in a teaching maternity unit. Br J Anaesth 1996, 76:680-684. | Article | PubMed

4. Barnardo PD, Jenkins JG: Failed tracheal intubation in obstetrics: a 6-year review in a UK region. Anaesthesia 2000, 55:690-694. | Article I PubMed

5. Cormack RS: Failed intubation in obstetric anaesthesia. Anaesthesia 2006, 61:505-506; author reply 506. | Article | PubMed

6. Djabatey EA, Barclay PM: Difficult and failed intubation in $\mathbf{3 4 3 0}$ obstetric general anaesthetics. Anaesthesia 2009, 64:1168-1171. | Article | PubMed

7. Rice T, Bowser C: Extrauterine abdominal pregnancy: report of a case. CRNA 1999, 10:181-183. | PubMed

8. Chantigian RC, Chantigan PD. Problems of early pregnancy. In: Chestnut DH, Polley LS, Tsen LC, Wong CA. Chestnut's Obstetric 
Kalava et al. Journal of Anesthesiology \& Clinical Science 2012, http://www.hoajonline.com/journals/pdf/2049-9752-1-13.pdf

Anesthesia Principles and Practice. 4th ed. 2009, 319-335.

Philadelphia, PA: Mosby Elsevier.

9. Rahman MS, Al-Suleiman SA, Rahman J, Al-Sibai MH: Advanced abdominal pregnancy--observations in 10 cases. Obstet Gynecol 1982, 59:366-372. | Article | PubMed

10. Costa SD, Presley J, Bastert G: Advanced abdominal pregnancy. Obstet Gynecol Surv 1991, 46:515-525. | Article | PubMed

11. Hughes $S$, Goodyear $P$, Sansome $A$ : The anaesthetic management of a woman with a 31-week abdominal pregnancy. Int J Obstet Anesth 2001, 10:321-324. | Article | PubMed

12. Martin JN, Jr., McCaul JFt: Emergent management of abdominal pregnancy. Clin Obstet Gynecol 1990, 33:438-447. | Article | PubMed

13. Roberts RV, Dickinson JE, Leung Y, Charles AK: Advanced abdominal pregnancy: still an occurrence in modern medicine. Aust $N \mathrm{Z} J$ Obstet Gynaecol 2005, 45:518-521. | Article | PubMed

14. Moodley J, Subrayen KT, Sankar D, Pitsoe SB: Advanced extra-uterine pregnancy associated with eclampsia. A report of 2 cases. S Afr Med J 1987, 71:460-461. | Article | PubMed

15. Worley KC, Hnat MD, Cunningham FG: Advanced extrauterine pregnancy: diagnostic and therapeutic challenges. Am J Obstet Gynecol 2008, 198:297 e291-297. | Article I PubMed

16.Stevens CA: Malformations and deformations in abdominal pregnancy. Am J Med Genet 1993, 47:1189-1195. | Article | PubMed

17.Ang LP, Tan AC, Yeo SH: Abdominal pregnancy: a case report and literature review. Singapore Med J 2000, 41:454-457. | Pdf | PubMed

18. Ramachandran K, Kirk P: Massive hemorrhage in a previously undiagnosed abdominal pregnancy presenting for elective Cesarean delivery. Can J Anaesth 2004, 51:57-61. | Article | PubMed

19. Hawkins JL, Koonin LM, Palmer SK, Gibbs CP: Anesthesia-related deaths during obstetric delivery in the United States, 1979-1990. Anesthesiology 1997, 86:277-284. | Article | PubMed

20. Kato R, Terui K, Yokota K, Watanabe M, Uokawa R, Miyao H. Anesthetic management for cases of placenta accreta presented for cesarean section: a 7-year single-center experience. Masui. 2008 Nov;57(11):1421-6. | Article | PubMed Abstract | PubMed Full Text

21. Kuczkowski KM: Anesthesia for the repeat cesarean section in the parturient with abnormal placentation: what does an obstetrician need to know? Arch Gynecol Obstet 2006, 273:319-321. | Article | PubMed

\section{Citation:}

Kalava A, Mardakh S, Weinberg J, Yarmush J, Schianodicola J and Ahmed K: Anesthetic management of an undiagnosed advanced extrauterine pregnancy under combined spinal-epidural anesthesia. journal of Anesthesiology and Clinical Science 2012, 1:13. http://dx.doi.org/10.7243/2049-9752-1-13 